Using ion beams to tune the nanostructure and optical response of co-deposited $\mathrm{Ag}: \mathrm{BN}$ thin films

This article has been downloaded from IOPscience. Please scroll down to see the full text article.

2007 J. Phys. D: Appl. Phys. 404614

(http://iopscience.iop.org/0022-3727/40/15/038)

View the table of contents for this issue, or go to the journal homepage for more

Download details:

IP Address: 161.111.22.141

The article was downloaded on 13/12/2012 at 11:24

Please note that terms and conditions apply. 


\title{
Using ion beams to tune the nanostructure and optical response of co-deposited Ag : BN thin films
}

\author{
J Toudert $^{1,3}$, D Babonneau ${ }^{1}$, S Camelio ${ }^{1}$, T Girardeau ${ }^{1}$, \\ F Yubero ${ }^{2}$, J P Espinós ${ }^{2}$ and A R Gonzalez-Elipe ${ }^{2}$ \\ ${ }^{1}$ Laboratoire de Métallurgie Physique, UMR CNRS 6630, Bâtiment SP2MI, \\ Boulevard Marie et Pierre Curie, Téléport 2 Futuroscope, 86962 Chasseneuil, France \\ ${ }^{2}$ Instituto de Ciencia de Materiales de Sevilla, CSIC, Isla de la Cartuja, \\ 41092 Isla de la Cartuja, Sevilla, Spain \\ E-mail: toudert@io.cfmac.csic.es
}

Received 3 April 2007, in final form 26 May 2007

Published 13 July 2007

Online at stacks.iop.org/JPhysD/40/4614

\begin{abstract}
The present study is devoted to co-deposited $\mathrm{Ag}$ : $\mathrm{BN}$ nanocermet thin films and is focused on the influence of ion irradiation conditions on their structural and linear optical properties. Ion irradiation was performed in situ during the growth of the nanocermets using a $50 \mathrm{eV}$ assistance beam (nitrogen/argon or nitrogen-ion assistance) and $e x$ situ on as-grown films using a $120 \mathrm{keV}$ argon-ion beam (post-irradiation). Grazing incidence small-angle $x$-ray scattering measurements show that (i) as-grown $\mathrm{N}$-assisted films contain prolate spheroidal clusters (height-to-diameter ratio $H / D \approx 1.8$ ), (ii) N/Ar-ion assistance leads to the formation of more elongated clusters $(H / D \approx 2.1)$ and (iii) post-irradiation leads to a decrease of $H / D$ to a value close to 1 . These results are discussed on the basis of atomic diffusion processes involved during the growth of the nanocermets and during the post-irradiation. The optical transmittance spectra of these films measured at normal incidence display one absorption band, due to the excitation of the $(1,1)$ plasmon mode of the clusters. In the case of the as-grown films, an additional band appears at oblique incidence for P-polarized light, as a consequence of the excitation of the $(1,0)$ plasmon mode of the clusters. Our results show that the spectral position of the absorption bands (which can be tuned in the $400-600 \mathrm{~nm}$ range) depends on the $H / D$ ratio of the clusters, in good agreement with calculations of optical transmittance considering the nanocomposite layer as a uniaxial anisotropic medium whose dielectric tensor is described by an anisotropic

Maxwell-Garnett model.
\end{abstract}

\section{Introduction}

During the past decades, a large number of studies have been devoted to nanoclusters, which are interesting building blocks for technological applications in chemistry [1], medical sciences [2,3] or material sciences [4-7]. Indeed, the small size of these clusters is responsible for their singular physical properties, which are different from those of the

3 Present address: Instituto de Optica, CSIC, c/Serrano 121, 28006 Madrid, Spain. bulk materials. In particular, noble metal nanoclusters exhibit a surface plasmon resonance (SPR) phenomenon, which is responsible for a strong absorption of light [8] and an enhancement of the electromagnetic field around the clusters in the visible range [9]. Free functionalized clusters can therefore be used as selective markers to increase the sensitivity of fluorescence biological analysis [10]. Nanoclusters can also be embedded within ceramic matrices to form composite materials. These materials, also called 'nanocermets', display a strong absorption band [8] and ultra-fast non-linear effects 
at the plasmon resonance [11], which make them potential candidates for the realization of opto-electronic components.

The linear and non-linear optical properties of nanocermets depend on the nature of their components, as well as the size, shape and organization of the clusters [8,12-15]. In order to synthesize materials with the desired properties, it is indeed of prime interest to control these parameters, which are related to the elaboration or post-treatment conditions of the material. Several chemical or physical techniques can be used to fabricate nanocermets $[11,15-21]$. In our previous studies [21-24], we showed that ion-beam sputtering can be used to get a significant control of the cluster morphology and spectral position of the SPR $(1,1)$ and $(1,0)$ modes of $\mathrm{Ag}: \mathrm{Si}_{3} \mathrm{~N}_{4}$ and $\mathrm{Ag}: \mathrm{BN}$ nanocermet thin films, these modes being excited for an incoming electric field oriented in the thin film plane or along its growth direction, respectively. Alternate deposition of the metal and of the dielectric species (dielectric/metal/dielectric trilayers) leads to the formation of metastable oblate clusters, whose height-to-diameter ratio $(H / D<1)$ can be adjusted by a proper choice of the amount of deposited metal (Ag), of the nature of the dielectric layers surrounding the clusters and of the duration of a delay before the deposition of the dielectric layer covering the clusters [21, 22]. The spectral position of the SPR $(1,1)$ mode of these trilayers has been shown to red-shift sensitively in a large wavelength range (from 400 to $800 \mathrm{~nm}$ ) when decreasing the height-to-diameter ratio of the clusters, in qualitative agreement with the predictions of the extended Maxwell-Garnett effective medium model [24]. In contrast, the co-deposition of silver and $\mathrm{Si}_{3} \mathrm{~N}_{4}$ leads to the formation of metastable prolate clusters (with $H / D>1$ ), exhibiting a SPR $(1,1)$ mode at a shorter wavelength than in the case of spherical clusters [24]. These results underline the necessity of controlling the growth kinetics of the metastable nanocermets in order to tailor their structure and therefore their optical response. Although some nice results concerning the control of the $H / D$ ratio of prolate noble metal clusters and of the spectral position of the SPR modes can be found in the literature in the case of thin films grown by physical techniques [25-28], only a few results have been reported up to now concerning nanocermets elaborated by co-deposition of metal and dielectric species. The aim of this paper is therefore to go further into the control of the morphology of Ag clusters embedded within a BN matrix using a co-deposition process. For this purpose, the influence of the nature of a low energy assistance ion-beam bombarding the films during the growth has been studied, because it is expected to play a significant role in the growth kinetics of the nanocermets and should consequently influence the morphology of the metastable clusters. Post-irradiation with higher energy argon ions has also been performed, in order to induce a reshaping of the metastable prolate clusters towards a (spherical) stable shape.

\section{Experimental details}

$\mathrm{BN} / \mathrm{Ag}$ : BN/BN nanocermets were grown at $473 \mathrm{~K}$ on fused silica and surface-oxidized silicon substrates by ion-beam sputtering in a Nordiko 3000 chamber. A primary $1200 \mathrm{eV}$ argon-ion beam was used to sputter alternatively a pure BN target and a 'home-made' composite target consisting of a BN target on which a silver wire was fixed. The deposition times were adjusted in order to obtain a $2 \mathrm{~nm}$ thick BN buffer, an effective thickness $t_{\mathrm{D}}=30 \mathrm{~nm}$ for the dielectric material constituting the $\mathrm{Ag}: \mathrm{BN}$ layer, and a $12 \mathrm{~nm}$ thick BN cap. A secondary $50 \mathrm{eV}$ nitrogen-ion beam was used to bombard the BN films during the deposition of the buffer and the cap, in order to obtain the correct $\mathrm{B}: \mathrm{N}$ stoichiometry by compensating for the $\mathrm{N}$ deficiency in the films. The effect of the assistance conditions has been studied by bombarding the $\mathrm{Ag}$ : $\mathrm{BN}$ films during the co-deposition process with a $50 \mathrm{eV}$ nitrogen-ion beam (N-assisted sample) or a $50 \mathrm{eV}$ nitrogen/argon-ion beam (N/Ar-assisted sample). To complete our study, the $\mathrm{N}$-assisted sample was post-irradiated at room temperature and at normal incidence with $120 \mathrm{keV}$ $\mathrm{Ar}^{+}$ions at a fluence of $5 \times 10^{15}$ ions $\mathrm{cm}^{-2}$. For such an incident kinetic energy, the SRIM code [29] indicates an almost uniform damage profile throughout the films and consequently a relatively homogeneous irradiated volume. The code also indicates that the $\mathrm{Ar}^{+}$incident ion profile is centred inside the substrate; therefore the $\mathrm{Ar}^{+}$concentration is very low in the nanocermet itself.

Silver effective thickness $t_{\mathrm{Ag}}$ in the as-grown $\mathrm{N}$-assisted and N/Ar-assisted films were determined by $\mathrm{x}$-ray fluorescence measurements from the $\mathrm{Ag} \mathrm{L}_{\alpha 1}$ and $\mathrm{Ag} \mathrm{L}_{\beta 1}$ peaks area, assuming the density of silver to be equal to the bulk one. Values of $3.0 \mathrm{~nm}$ and $2.8 \mathrm{~nm}$ were obtained, respectively, suggesting that no significant silver re-sputtering occurs in the case of the N/Ar-assisted film. Silver volume fraction $f_{\mathrm{Ag}}$ was determined using the relation $f_{\mathrm{Ag}}=t_{\mathrm{Ag}} /\left(t_{\mathrm{Ag}}+t_{\mathrm{D}}\right)$ leading to $9.2 \%$ in the case of the $\mathrm{N}$-assisted sample and $8.5 \%$ for the $\mathrm{N} /$ Ar-assisted one. Let us note that $f_{\mathrm{Ag}}$ remains lower than $10 \%$ in each case; the samples studied here should therefore contain diluted systems of clusters.

The morphology and organization of the clusters were studied by grazing incidence small angle $\mathrm{x}$-ray scattering (GISAXS) measurements carried out at the European Synchrotron Radiation Facility (ESRF, Grenoble, France) on beam-line D2AM. The energy of the incident photons was set to $8 \mathrm{keV}$ while the beam size was $\sim 0.5 \times 0.1 \mathrm{~mm}^{2}$. Good surface sensitivity was achieved by working under an angle of incidence $\alpha_{\mathrm{i}}$ slightly above the critical angle for total reflection $\left(\alpha_{\mathrm{c}} \approx 0.3^{\circ}\right)$, so that the penetration depth of the $\mathrm{x}$-ray beam was of the order of the film thickness. The transmitted and specularly reflected beams were masked by a vertical beamstop and the scattered radiation was collected on a 2D CCD detector placed at $530 \mathrm{~mm}$ behind the sample. Before analysis, the scattering patterns were corrected for refraction, absorption and transmission effects [25] in the framework of the distortedwave Born approximation (DWBA) [30].

The optical properties of the thin films were determined by optical transmittance measurements carried out at normal and oblique incidence with a polarized light, between 250 and $800 \mathrm{~nm}$ with a $10 \mathrm{~nm}$ monochromator step.

\section{Results and discussion}

\subsection{Morphology and organization of the clusters}

GISAXS patterns of the as-grown N-assisted and N/Ar-assisted samples and of the post-irradiated $\mathrm{N}$-assisted sample are presented in figures $1(a), 2(a)$ and 3(a), respectively. 

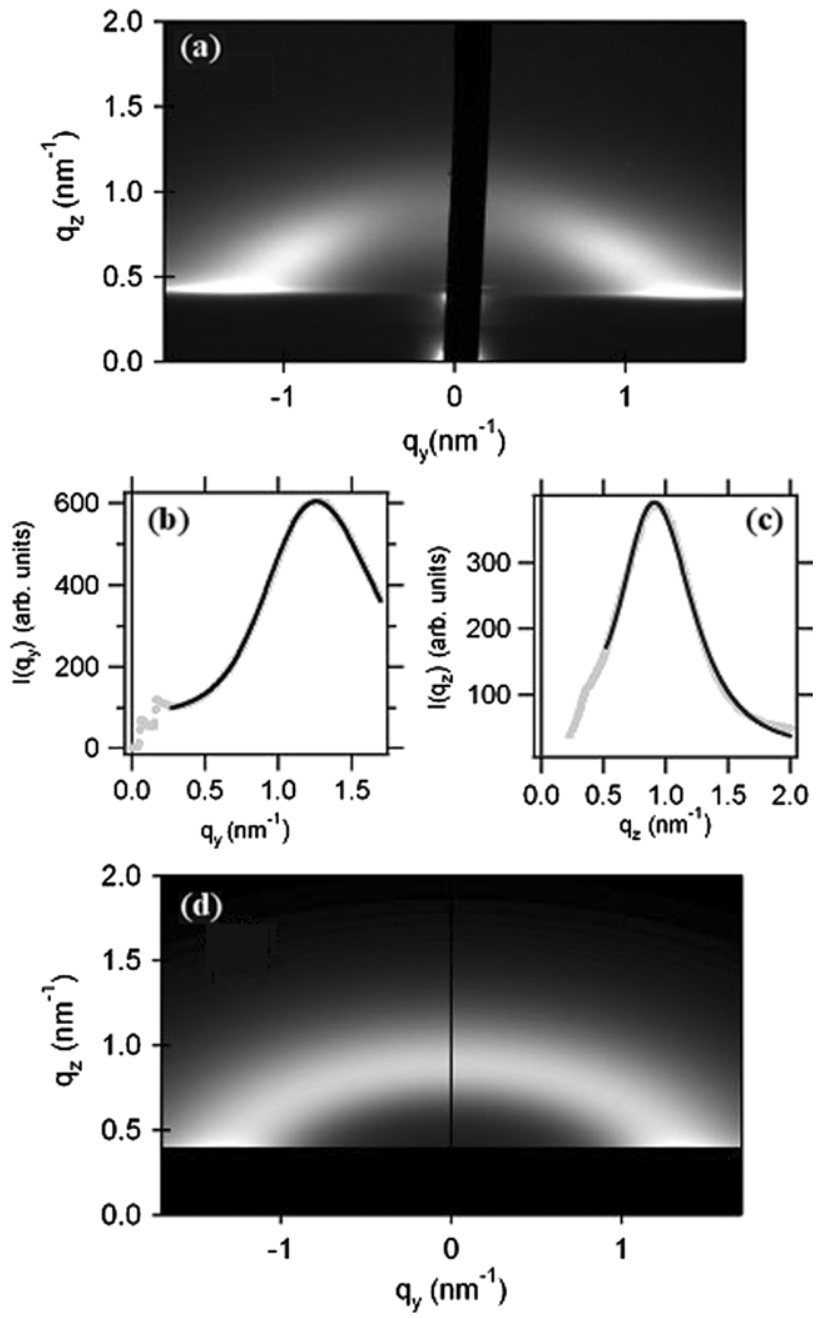

Figure 1. (a) GISAXS pattern of the as-grown $\mathrm{N}$-assisted film. Measured scattered intensity (grey dots) and best fit (black line): (b) along the horizontal axis, (c) along the vertical axis.

(d) Calculated GISAXS pattern from the best fit values of the parameters.

Let us first note that, in the case of as-grown films, no intense scattering can be seen close to the origin of the reciprocal space, in contrast to the results obtained in the case of uncapped $\mathrm{Ag}: \mathrm{Si}_{3} \mathrm{~N}_{4}$ and $\mathrm{Ag}$ :BN co-sputtered thin films studied in previous papers $[23,24,31]$. This intense scattering was related to the presence of large clusters supported by the co-sputtered films, which were formed by diffusion of silver atoms towards the surface. As can be seen here, this phenomenon can be avoided by depositing a dielectric cap onto the co-sputtered layer immediately after the end of the co-deposition process. GISAXS patterns of uncapped $\mathrm{Ag}: \mathrm{Si}_{3} \mathrm{~N}_{4}$ and $\mathrm{Ag}: \mathrm{BN}$ co-sputtered thin films also displayed intensity maxima for larger $q$ values, which were attributed to $\mathrm{x}$-ray scattering events by smaller clusters dispersed within the co-deposited layer. Such features can be seen here in the three GISAXS patterns, confirming the presence of nanoclusters inside the films. In the case of as-grown samples (figures $1(a)$ and $2(a)$ ), one can observe an elliptic ring elongated in the $q_{y}$ direction, linked to the presence of spatially correlated spheroidal prolate clusters within the matrix [31,32], presenting strong vertical and horizontal correlation. This ring
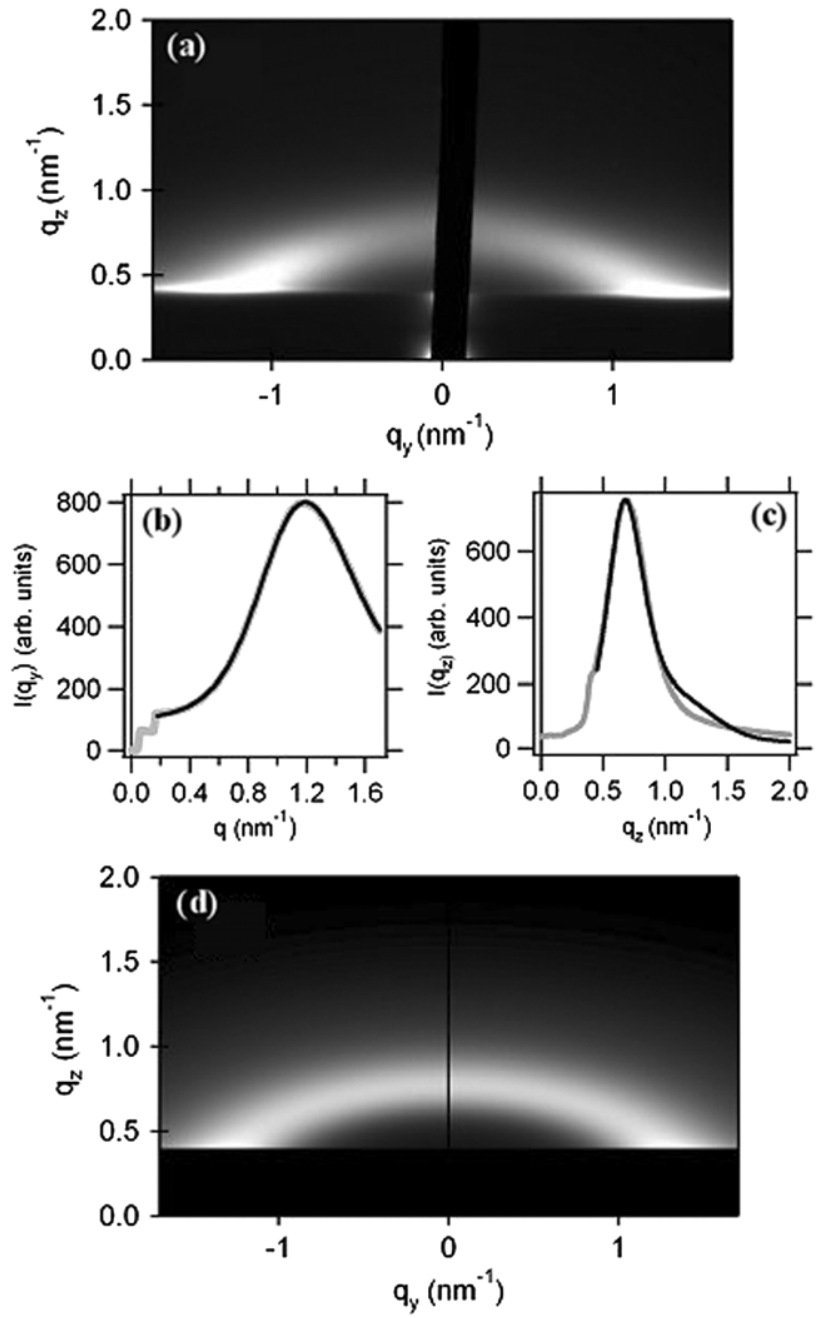

Figure 2. (a) GISAXS pattern of the as-grown N/Ar-assisted film. Measured scattered intensity (grey dots) and best fit (black line): (b) along the horizontal axis, (c) along the vertical axis.

(d) Calculated GISAXS pattern from the best fit values of the parameters.

is flatter in the case of a N/Ar assistance (figure 2(a)) than in the case of a $\mathrm{N}$ assistance (figure 1(a)), suggesting that the former leads to the formation of more prolate clusters than the latter. The GISAXS pattern of the post-irradiated $\mathrm{N}$-assisted sample (figure 3(a)) also displays an elliptic scattering ring, which is, however, less elongated along the $q_{y}$ direction than in the case of the as-grown samples, suggesting that the shape anisotropy of the clusters is reduced because of ion irradiation [32].

Quantitative information about the morphology and inplane organization of the clusters has been obtained from an adjustment of simulated x-ray scattering profiles to the experimental ones along the two directions (close to the axis of the elliptic rings, one being parallel to $q_{y}$ and the other one to $q_{z}$ ) according to the method described in [25]. The simulations were performed in the framework of the local monodisperse approximation (LMA) [33], assuming a lognormal size distribution for the nanoclusters, and using a spheroidal form factor to account for the cluster morphology. The organization of the clusters was assumed to be correctly described by a hard sphere structure factor [33]: the size of the 'spheroidal' hard spheres was supposed to be proportional 

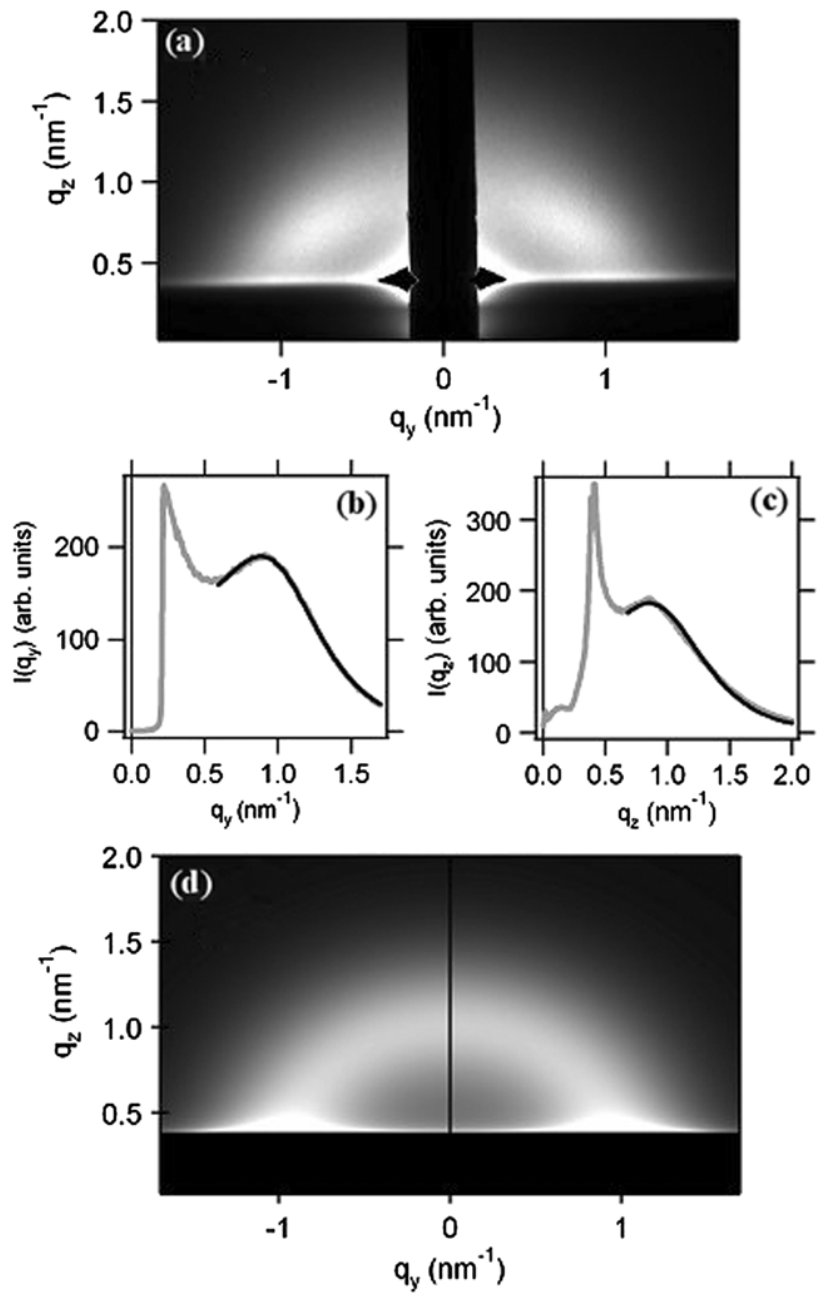

Figure 3. (a) GISAXS pattern of the post-irradiated N-assisted film. Measured scattered intensity (grey dots) and best fit (black line): (b) along the horizontal axis, (c) along the vertical axis. (d) Calculated GISAXS pattern from the best fit values of the parameters.

to the size of the clusters with a filling factor $C(>1)$, and the spatial disorder was represented by their volume fraction $\eta$. The fit parameters were the cluster in-plane diameter $D$, the half width at half maximum (HWHM) of the $D$ distribution, the height-to-diameter ratio $H / D$, the hard sphere compacity $\eta$ and the filling factor $C$. Since strong vertical and horizontal spatial correlation have been evidenced in the case of all samples, the values of $\eta$ and $C$ were assumed to be the same in the horizontal plane and in the growth direction. The experimental profiles and best fit curves are displayed in figures $1(b)-(c), 2(b)-(c)$ and $3(b)-(c)$, and the corresponding GISAXS patterns calculated from the best fit values of the parameters are shown in figures $1(d), 2(d)$ and $3(d)$. These values are gathered in table 1 , as well as the average in-plane intercluster distance $\Lambda_{x y}$, determined from the position $q_{\max }$ of the maximum of the structure factor (calculated using $\eta$ and $C$ values [33]), following the relation $\Lambda_{x y}=2 \pi / q_{\max }$.

In the case of the as-grown samples, quantitative analysis confirms the prolate shape of the clusters, which can be explained on the basis of thermodynamics and growth kinetics
Table 1. Structural parameters extracted from the quantitative analysis of the GISAXS patterns using the local monodisperse approximation.

\begin{tabular}{lllllll}
\hline Sample & $\begin{array}{l}D \\
(\mathrm{~nm})\end{array}$ & $\begin{array}{l}\mathrm{w} \\
(\mathrm{nm})\end{array}$ & $C$ & $\eta$ & $\begin{array}{l}\Lambda_{x y} \\
(\mathrm{~nm})\end{array}$ & $H / D$ \\
\hline N assisted (as-grown) & 2.2 & 0.9 & 1.7 & 0.38 & 3.9 & 1.8 \\
N/Ar assisted (as-grown) & 2.1 & 0.7 & 2.0 & 0.39 & 4.7 & 2.1 \\
N assisted (post-irradiated) & 3.4 & 1.1 & 1.4 & 0.24 & 5.2 & 1.1 \\
\hline
\end{tabular}

of the deposited films. In the present case, sputtered species coming from the targets towards the substrates present a low kinetic energy (in the $1-10 \mathrm{eV}$ range). Growth is therefore dominated by diffusion processes of the deposited species at the surface of the film. During the early stages of the codeposited layer growth, surface diffusion and aggregation of silver atoms on the buffer layer lead to the formation of silver nuclei following a Volmer-Weber-like growth mechanism [1]. Then, because of the low value of the ratio between deposition rates of silver and dielectric material, the space between growing silver clusters is quickly filled with dielectric species. Consequently, silver atoms incoming onto the growing clusters are more likely trapped by their top face than by their lateral faces, leading to a columnar growth [25]. Let us note that trapping of deposited silver atoms (presenting a too low kinetic energy) at the surface of the dielectric material between columnar clusters is also possible and leads to the formation of new silver nuclei to the detriment of the columnar growth of already existing clusters. The efficiency of the columnar growth mechanism depends therefore on the fraction of deposited silver atoms trapped by the growing columnar clusters.

Quantitative analysis of the GISAXS patterns also shows that the addition of argon ions to the nitrogen assistance beam leads to an increase in the height-to-diameter ratio and in-plane separation of the clusters, and to a narrowing of the diameter distribution. These results underline the crucial influence of the $50 \mathrm{eV}$ assistance beam on the growth of the cosputtered $\mathrm{Ag}$ : BN layers: heavy species (such as argon) enables an enhancement of the energy transfer from the assistance beam to the growing layer surface [25]. Surface diffusion of deposited silver atoms is consequently increased. The capture of these atoms by the top face of growing columnar clusters is therefore facilitated to the detriment of the formation of new nuclei, leading to an increase in $H / D$ and $\Lambda_{x y}$, as is effectively observed.

It can also be seen from table 1 that post-irradiation leads to an increase in the cluster size and in-plane inter-cluster separation, and to a decrease in the height-to-diameter ratio of the clusters. The energy of the post-irradiation $\mathrm{Ar}^{+}$ion beam $(120 \mathrm{keV})$ being two orders of magnitude higher than the kinetic energy of species involved during the growth of as-prepared samples, volume rearrangements are more likely to occur than surface events. The increase in cluster diameter and in-plane separation induced by ion post-irradiation could therefore result either from silver atom volume diffusion or volume coalescence events induced by the energy transfer from incoming $\mathrm{Ar}^{+}$ions to the irradiated material. This energy transfer also allows a reshaping of the clusters, from a prolate and metastable shape to a quasi spherical shape, thus 

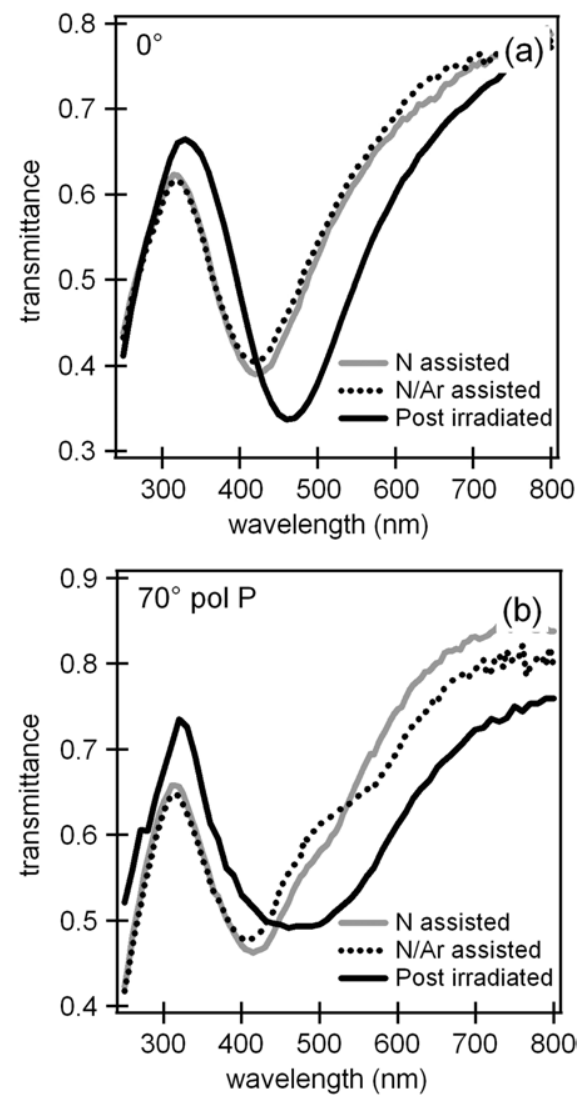

Figure 4. Optical transmittance spectra of as-grown $\mathrm{N}$ assisted film (grey lines), as-grown N/Ar assisted film (black dotted lines) and post-irradiated $\mathrm{N}$-assisted sample (black lines), (a) at normal incidence, $(b)$ at oblique incidence $\left(70^{\circ}\right)$ with a P-polarized light.

minimizing the free energy of the cluster/surrounding matrix system.

\subsection{Optical properties}

The optical transmittance spectra of the films recorded at normal and oblique incidence $\left(70^{\circ}\right.$, P-polarized light) are presented in figure 4 . These spectra display absorption bands commonly associated with plasmon resonances within the clusters. According to the predictions of the extended Maxwell-Garnett effective medium model [23], which is theoretically adequate for dilute tri-dimensional assemblies of nanoclusters such as the ones encountered in this study, the spectral position of the SPRs depend on the nature of the metal and of the matrix, and on the volume fraction and shape of the clusters. Since the silver volume fraction displays similar values in all the films under study, the evolution of the spectral positions of the absorption bands as a function of ion irradiation conditions can therefore be discussed as a shape effect.

At normal incidence (figure 4(a)), one can observe one absorption band due to the plasmon resonance mode of the clusters resulting from their excitation by an electric field perpendicular to the growth direction of the film $((1,1)$ mode). While assistance conditions have no influence on the spectral position of this band, post-irradiation of the $\mathrm{N}$-assisted sample (which induces a decrease of $H / D$ from 1.8 to 1.1) leads to a strong shift of this band towards longer wavelength.
This behaviour is in qualitative agreement with theoretical calculations assuming isolated clusters [8], which show that the wavelength of the $(1,1)$ mode of spheroidal clusters is a decreasing function of $H / D$ with a horizontal asymptotic behaviour for high $H / D$ values.

At oblique incidence and for a P-polarized light (figure $4(b)$ ), an additional band appears at a longer wavelength than the one associated with the $(1,1)$ mode in the case of asgrown films. This band is related to the plasmon resonance mode of the clusters resulting from their excitation by an electric field oriented along the growth direction of the film $((1,0)$ mode $)$, and appears at a longer wavelength in the case of the N/Ar-assisted sample. These results are qualitatively consistent with theoretical calculations [8] which show that the $(1,0)$ mode spectral position is an increasing function of $H / D$ and that this mode is red-shifted from the $(1,1)$ one in the case of prolate clusters. In the case of spherical clusters, both modes coincide, and only one band is expected at oblique incidence, as effectively observed for the post-irradiated sample containing quasi-spherical clusters. Let us however note that the broad band observed for this film might be the envelope of two bands located at wavelengths one close to another.

In order to go further into the study of the optical response of the present thin films, we investigated the possibility of calculating accurately the spectral position of their absorption bands, using an adequate model and structural data extracted from the GISAXS analysis. For this purpose, we considered each sample as a layered system using the following sequence: $\mathrm{SiO}_{2}$ substrate/BN buffer/Ag: BN nanocomposite layer/BN cap. The optical properties of the substrate and of the BN layers were described by their dielectric functions $\left(\varepsilon_{\mathrm{SiO}_{2}}=2.25\right.$ and $\varepsilon_{\mathrm{BN}}=3.24$ ), which were measured by spectroscopic ellipsometry. The optical properties of the anisotropic Ag: BN layer were described by a dielectric diagonal tensor whose components $\varepsilon_{u}$ were the dielectric functions of the material for an in-plane excitation $\left(\varepsilon_{x y}\right)$ and for an excitation along the growth direction $\left(\varepsilon_{z}\right)$. These dielectric functions were calculated using the extended Maxwell-Garnett model:

$\varepsilon_{u}=\varepsilon_{\mathrm{BN}}+f \frac{\varepsilon_{\mathrm{BN}}\left(\varepsilon_{\mathrm{i}}-\varepsilon_{\mathrm{BN}}\right)}{\varepsilon_{\mathrm{BN}}+\left(L_{u}-\frac{f}{3}\right)\left(\varepsilon_{\mathrm{i}}-\varepsilon_{\mathrm{BN}}\right)}, \quad$ with $u=x y$ or $z$,

where the volume fraction $f$ of silver was set to $9 \%$, according to x-ray fluorescence results, the dielectric function $\varepsilon_{\mathrm{i}}$ of the metal clusters was assumed to be the one of bulk silver [34], and the values of the depolarization factors $L_{x y}$ and $L_{z}$ [35] were calculated from the $H / D$ values determined by GISAXS.

Figure 5 regroups the refractive indices $n_{u}$ and extinction coefficients $k_{u}$ of the nanocomposite layer calculated in the case of the Ar/ $\mathrm{N}$ assisted sample, following the relation $\varepsilon_{u}=$ $\left(n_{u}+j k_{u}\right)^{2}$. The maximum of $k_{x y}$, i.e. the $(1,1)$ plasmon mode, is observed at $410 \mathrm{~nm}$, close to the spectral position of the absorption band observed by transmittance measurements at normal incidence. A quite different behaviour is evidenced for the $(1,0)$ plasmon mode, corresponding to the maximum of $k_{z}$ located at $690 \mathrm{~nm}$, i.e. at a significantly longer wavelength than the second band revealed by transmittance measurements at oblique incidence with a $\mathrm{P}$ polarized light, which is located at $560 \mathrm{~nm}$. This striking difference, also observed by Atkinson et al in the case of Au nanorods embedded in $\mathrm{Al}_{2} \mathrm{O}_{3}$ [26], has 


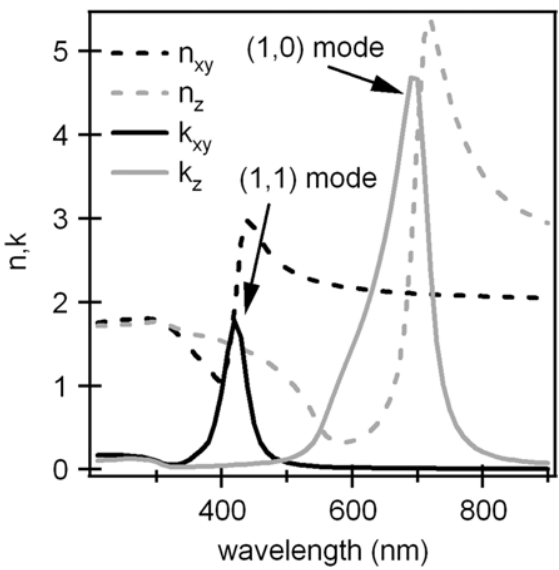

Figure 5. Calculated refractive index $n$ and extinction coefficient $k$ in the horizontal plane $(x y)$ and along the vertical direction $(z)$, using the extended Maxwell-Garnett model with GISAXS parameters of N/Ar assisted sample, $f=9 \%$, and assuming bulk properties for silver.
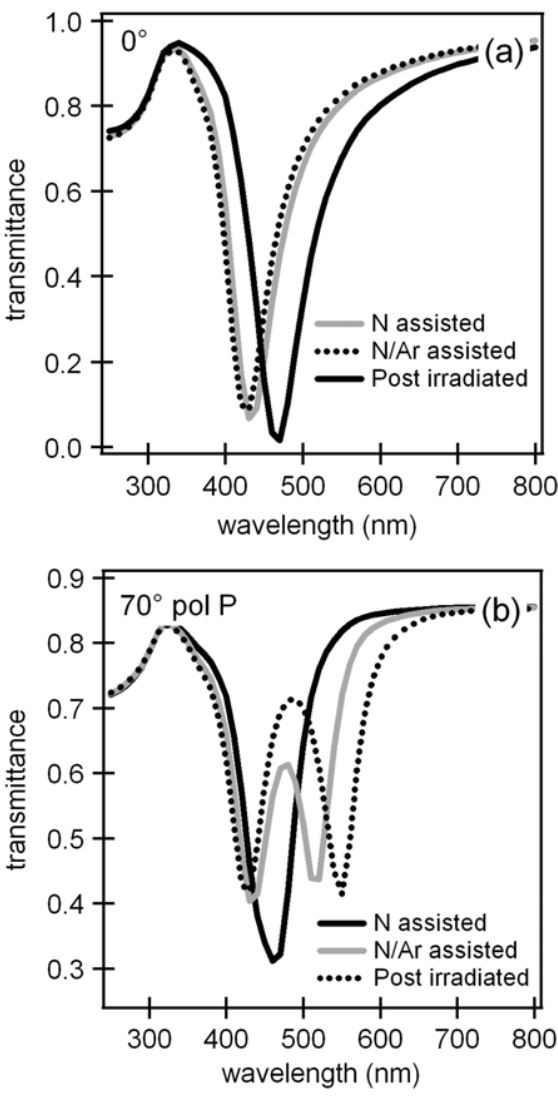

Figure 6. Calculated optical transmittance spectra of as-grown $\mathrm{N}$-assisted film (grey lines), as-grown N/Ar-assisted film (black dotted lines) and post-irradiated $\mathrm{N}$-assisted sample (black lines), $(a)$ at normal incidence, $(b)$ at oblique incidence $\left(70^{\circ}\right)$ with a P-polarized light. An anisotropic multilayer model was used, the dielectric tensor of the nanocomposite layer being described by the extended Maxwell-Garnett model. The dielectric function used for silver was the bulk one.

two origins. On the one hand, optical transmittance depends on absorption but also refraction effects. On the other hand, the optical properties of the present anisotropic medium at oblique incidence do not result from a simple linear combination of the

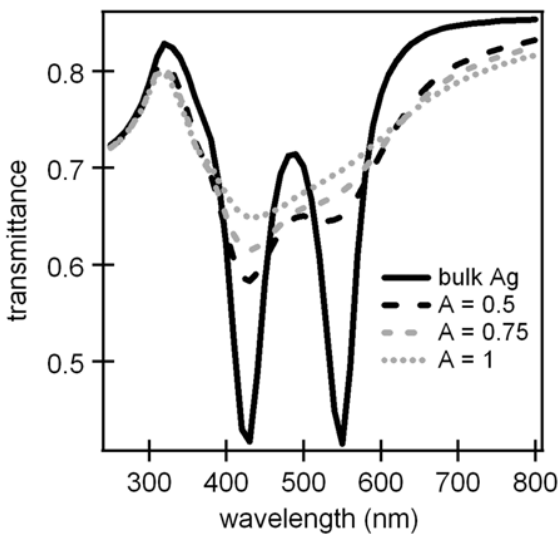

Figure 7. Calculated optical transmittance spectra of as-grown N/Ar-assisted film at oblique incidence $\left(70^{\circ}\right)$ with a P-polarized light, using a modified silver dielectric function in order to take into account finite size effects of the optical properties of the clusters. The collision frequency in the modified dielectric function was calculated by adding a $A v_{\mathrm{F}} / R$ term to the bulk value, $A$ being an adjustable parameter ranging from 0 (bulk properties for $\mathrm{Ag}$ ) to 1 .

dielectric functions corresponding respectively to an excitation in the horizontal plane and along the growth direction of the film [26], the extraordinary index surface displaying a spheroidal shape in the present case [36]. For these reasons, the second band appearing at oblique incidence is not exactly the ' $(1,0)$ plasmon absorption band', even if its existence is linked to the excitation of the $(1,0)$ plasmon mode. This mode, even if excited, is optically inactive, in the sense that no extinction band can be observed at $690 \mathrm{~nm}$.

For these reasons, the prediction of the spectral position of both absorption bands requires the calculation of the transmittance of the layered system. The optical transmittance at normal and oblique incidence $\left(70^{\circ}\right)$ with a P-polarized light were calculated for each system, taking into account light propagation within the layers, reflexion and refraction effects at interfaces. The spectral positions of the absorption bands observed in the simulated spectra-presented in figure 6-are in good agreement with the experimental results.

The spectral position of the bands observed in the transmittance spectra of the thin films under study are linked to the shape of the nanoclusters, as determined by GISAXS. As mentioned by Atkinson et al [26], the extraction of quantitative morphological data from optical transmittance spectra however requires to describe correctly the anisotropic behaviour of the nanocomposite material, which is to our knowledge not always the case in the literature.

Strong discrepancies are however evidenced concerning bandwidths and amplitudes, probably because the model used here does not take into account the influence of the nanometric finite size of the clusters on silver dielectric function. A simple way to estimate the influence of this size effect consists of correcting the collision frequency of conduction electrons in the metal, that is increased because of interactions with the surface of the cluster [8]. The correcting term follows a $A v_{\mathrm{F}} / R$ law, where $R$ is the effective radius of the cluster, $v_{\mathrm{F}}$ the Fermi velocity of silver and $A$ an adjustable parameter, which reflects the intensity of size effects and depends on the nature of the cluster/matrix interface. Such size effects are known to induce an attenuation and a spectral broadening of 
the plasmon absorption bands and to have no effect on their spectral position [8], as is observed in figure 7 in the case of the N/Ar assisted sample.

\section{Conclusions}

The co-sputtering of silver and BN leads to the formation of metastable prolate spheroidal silver nanoclusters (height to diameter ratio $H / D>1$ ) following a columnar growth mechanism, that can be more or less efficient depending on the diffusion of silver atoms at the surface of the growing film. Surface diffusion processes can be enhanced by improving the energy transfer from the $50 \mathrm{eV}$ ion assistance beam to surface silver atoms. For example, adding heavier ions to the assistance beam increases surface diffusion processes and leads to the formation of clusters with a higher $H / D$ ratio. $H / D$ has been varied from 1.8 to 2.1 as a function of assistance conditions. A lower $H / D$ value of 1.1 has been obtained after post-irradiation of as-prepared samples with a $120 \mathrm{keV} \mathrm{Ar}^{+}$ ion beam, the decrease in $H / D$ resulting from a silver volume diffusion-induced reshaping of the clusters from a metastable prolate shape to a stable spherical shape.

The optical transmittance spectra of these films at normal incidence display one absorption band, due to the excitation of the $(1,1)$ plasmon mode of the clusters. In the case of asgrown films, an additional band appears at oblique incidence for a P-polarized light, as a consequence of the excitation of the $(1,1)$ and $(1,0)$ plasmon modes, which appear at different wavelengths for anisotropic clusters. No second band can be clearly evidenced in the case of the post-irradiated sample, containing quasi-spherical clusters, for which the $(1,1)$ and the $(1,0)$ modes occur nearly at the same wavelength. The spectral position of the absorption bands of these films (tuned in the $400-600 \mathrm{~nm}$ range) are in good agreement with the calculations of optical transmittance considering the nanocomposite layer as a uniaxially anisotropic medium which dielectric tensor is described by an anisotropic Maxwell-Garnett model. These results moreover show that a proper description of anisotropy is mandatory for extracting quantitative information concerning cluster shape from optical transmittance spectra at oblique incidence.

\section{Acknowledgments}

The authors would like to thank CNRS-CSIC and Picasso programmes for financial support which permitted the collaboration between the Instituto de Ciencia de Materiales de Sevilla (Spain) and the Laboratoire de Métallurgie Physique de Poitiers (France). The authors also thank J P Simon and the D2AM staff at the ESRF for their support during the GISAXS measurements.

\section{References}

[1] Henry C R 1998 Surf. Sci. Rep. 31 231-325

[2] Schalkammer T, Leitner A, Aussenegg F R, Bauer G and Pittner F 1998 Proc. SPIE 3253 12-19
[3] Zharov V P, Galitovskaya E N, Johnson C and Kelly T 2005 Lasers Surg. Med. 37 219-26

[4] Weiss N et al 2005 Phys. Rev. Lett. 95157204

[5] Barner J P and Ruggiero S T 1987 Phys. Rev. Lett. 59 $807-10$

[6] Ung T, Liz-Marzan L M and Mulvaney P 2002 Colloids Surf. A 202 119-26

[7] Pinçon N, Palpant B, Prot D, Charron E and Debrus S 2002 Eur. Phys. J. D 19 395-402

[8] Kreibig U and Vollmer M 1995 Optical Properties of Metal Clusters (Berlin: Springer)

[9] Quidant R, Girard C, Weeber J C and Dereux A 2004 Phys. Rev. B 69085407

[10] Alschinger M, Maniak M, Stietz F, Vartanyan T and Träger F 2003 Appl. Phys. B 76 771-4

[11] Del Coso R, Requejo-Isodro J, Solis J, Gonzalo J and Afonso C N 2004 J. Appl. Phys. 95 2755-62

[12] Noguez C 2005 Opt. Mater. 27 1204-11

[13] Noguez C and Barrera R G 1998 Phys. Rev. B 57 302-13

[14] Palpant B, Prével B, Lermé J, Cottancin E, Pellarin M, Treilleux M, Perez A, Vialle J L and Broyer M 1998 Phys. Rev. B 57 1963-70

[15] Lee M, Kim T S and Choi Y S 1997 J. Non-Cryst. Solids 211 143-9

[16] Gonella F 2000 Nucl. Instrum. Methods B 166 831-9

[17] Zuhr R A, Magruder III R H and Anderson T S 1998 Surf. Coat. Technol. 103-104 401-8

[18] Battaglin G, Cataruzza E, Gonella F, Mattei G, Mazzoldi P, Sada C and Zhang X 2000 Nucl. Instrum. Methods B $166857-63$

[19] Perez A et al 1997 J. Phys. D: Appl. Phys. 30 709-21

[20] Barnes J P, Petford-Long A K, Doole R C, Serna R, Gonzalo J, Suarez-Garcia A, Afonso C N and Hole D 2002 Nanotechnology 13 465-70

[21] Toudert J, Camelio S, Babonneau D, Denanot M F, Girardeau T, Espiños J P, Yubero F and Gonzalez-Elipe A R 2006 J. Appl. Phys. 98114316

[22] Toudert J, Camelio S, Babonneau D and Girardeau T 2005 Rev. Adv. Mater. Sci. 10 123-7

[23] Camelio S, Babonneau D, Girardeau T, Toudert J, Lignou F, Denanot M F, Maître N, Barranco A and Guérin P 2003 Appl. Opt. 42 674-81

[24] Camelio S, Toudert J, Babonneau D and Girardeau T 2005 Appl. Phys. B 80 89-96

[25] Babonneau D, Pailloux F, Eymery J P, Denanot M F, Guérin P, Fonda E and Lyon O 2005 Phys. Rev. B 71035430

[26] Atkinson R, Hendren W R, Wurtz G A, Dickson W, Zayats A V, Evans P and Pollard R J 2006 Phys. Rev. B $\mathbf{7 3} 235402$

[27] Margueritat J, Gonzalo J, Afonso C N, Ortiz M I and Ballesteros C 2006 Appl. Phys. Lett. 88093107

[28] Margueritat J, Gonzalo J, Afonso C N, Mlayah A, Murray D B and Saviot L 2006 Nanolett. 6 2037-42

[29] Ziegler J F and Biersack J P 1985 The Stopping and Range of Ions in Solids (Oxford: Pergamon)

[30] Rauscher M, Salditt T and Spohn H 1995 Phys. Rev. B 5216855

[31] Babonneau D, Camelio S, Girardeau T, Jaouen M and Lyon O 2003 J. Appl. Crystallogr. 36 430-4

[32] Babonneau D, Naudon A, Cabioc'h T and Lyon O 2000 J. Appl. Crystallogr. 33 437-41

[33] Pedersen J S 1994 J. Appl. Crystallogr. 27 595-608

[34] Palik E D 1985 Handbook of Optical Constants(New York: Academic)

[35] Dakka A, Lafait J, Sella C, Berthier S, Abd-Lefdil M, Martin J C and Maaza M 2000 Appl. Opt. 39 2745-53

[36] Born M and Wolf E 1959 Principles of Optics (Oxford: Pergamon) 